\title{
BMJ Open Serum 25-hydroxyvitamin D levels in relation to lipids and clinical outcomes in pregnant women with gestational diabetes mellitus: an observational cohort study
}

Wenqing Chen, ${ }^{1}$ Yuan $\mathrm{Li}^{1}$, Bo Gao, ${ }^{1} \mathrm{Jie} \mathrm{Li},{ }^{2}$ Mingming Zheng, ${ }^{2}$ Xiaotian Chen (D) ${ }^{1}$

To cite: Chen W, Li Y, Gao B, et al. Serum 25-hydroxyvitamin $D$ levels in relation to lipids and clinical outcomes in pregnant women with gestational diabetes mellitus: an observational cohort study. BMJ Open 2020;10:e039905. doi:10.1136/ bmjopen-2020-039905

- Prepublication history and additional material for this paper are available online. To view these files, please visit the journal online (http://dx.doi. org/10.1136/bmjopen-2020039905).

Received 29 April 2020 Revised 28 0ctober 2020 Accepted 29 0ctober 2020
Check for updates

(c) Author(s) (or their employer(s)) 2020. Re-use permitted under CC BY-NC. No commercial re-use. See rights and permissions. Published by BMJ.

1Department of Clinical Nutrition, Nanjing Drum Tower Hospital, Nanjing, China

${ }^{2}$ Department of Obstetrics and Gynecology, Nanjing Drum Tower Hospital, Nanjing, China

Correspondence to

Dr Xiaotian Chen;

xttchen@163.com

\section{ABSTRACT}

Objective The aim of the present study was to investigate whether 25-hydroxyvitamin D (25(OH)D) status at 24-28 weeks is associated with blood lipids and pregnancy outcomes in patients with gestational diabetes mellitus (GDM).

Design We performed an observational cohort study. Setting The study was conducted in China.

Participants A total of 261 pregnant women diagnosed with GDM at 24-28 weeks of gestation in our hospital were included between June 2015 and December 2017. According to the levels of $25(\mathrm{OH}) \mathrm{D}$, the women were divided into the $\mathrm{G} 1(<20 \mathrm{ng} / \mathrm{mL})$ and $\mathrm{G} 2(\geq 20 \mathrm{ng} / \mathrm{mL})$ groups. The levels of total cholesterol (TC), triglyceride (TG), high-density lipoprotein cholesterol (HDL-C), lowdensity lipoprotein cholesterol (LDL-C), TG/HDL-C and TC/HDL-C ratios were obtained from medical records. Pregnancy outcomes included gestational weeks of birth and delivery mode. Newborn information included birth weight and body length. Differences between groups were tested with adjusted multiple linear regression.

Results The serum levels of $25(\mathrm{OH}) \mathrm{D}(14.1 \pm 3.4 \mathrm{ng} / \mathrm{mL}$ vs $28.5 \pm 6.5 \mathrm{ng} / \mathrm{mL}, p<0.001)$, TC $(5.3 \pm 0.9$ vs $5.6 \pm 0.8$, $\mathrm{p}=0.006)$, HDL-c ( $1.8 \pm 0.4$ vs $1.9 \pm 0.4, p=0.046)$ and LDL-C $(2.5 \pm 0.6$ vs $2.7 \pm 0.7, p=0.015)$ in the G2 group were significantly higher than those in G1 group, while $\mathrm{TG} / \mathrm{HDL}-\mathrm{c}$ ratios $(1.43 \pm 0.7$ vs $1.26 \pm 0.7, \mathrm{p}=0.035)$ were significantly higher in the G1 group. Moreover, we failed to find a significant difference in pregnancy outcomes of mothers and newborns among the two groups $(p>0.05)$. In models adjusting for maternal age, parity, height, blood pressure, socioeconomic status, educational attainment, pre-pregnancy body mass index, season and gestational age, maternal $25(\mathrm{OH}) \mathrm{D}$ was associated with $\mathrm{TG} / \mathrm{HDL}-\mathrm{C}$ ratios $(\mathrm{B}=-0.016 ; 95 \% \mathrm{Cl}=-0.025$ to -0.006$)$.

Conclusion We found that there was no relationship between vitamin $D$ and pregnancy/neonatal outcomes in our study. Maternal $25(\mathrm{OH}) \mathrm{D}$ at 24-28 weeks was inversely associated with $\mathrm{TG} / \mathrm{HDL}-\mathrm{c}$ ratios.

\section{INTRODUCTION}

Gestational diabetes mellitus (GDM) refers to different degrees of abnormal glucose metabolism first occurring or first identified
Strengths and limitations of this study

To the best of our knowledge, this is the first study to evaluate the association between 25 -hydroxyvitamin D $(25(\mathrm{OH}) \mathrm{D})$ concentrations and lipid profile during pregnancy in a Chinese gestational diabetes mellitus (GDM) population based on the grouping of vitamin $D$ levels.

- In this study, we designed a questionnaire containing clinically relevant factors, including age, body mass index, parity and seasons for women at different educational attainment.

- Serum 25(OH)D was measured after women were diagnosed with GDM, which enabled the assessment of longitudinal relationships between $25(\mathrm{OH})$ $\mathrm{D}$ and pregnancy outcomes in the GDM population.

- Confounding factors were the main limitations of this retrospective observational design.

- The cohort of women with GDM may not represent the general population of women with GDM due to the location of subject recruitment and small sample size.

in pregnancy, and it is one of the common complications during pregnancy. The total prevalence of GDM in China is $14.8 \% .^{1}$ Because of the adaptations to pregnancy, pregnant women will have some physiological changes in lipid metabolism. ${ }^{2}$

Vitamin D is a necessary fat-soluble vitamin for the human body; it is involved not only in regulating calcium and phosphorus metabolism, but also in improving insulin resistance, regulating insulin secretion and blood lipid metabolism. $^{3-5}$ Studies have shown that vitamin $\mathrm{D}$ deficiency may be associated with increased risk of GDM and dyslipidaemia. ${ }^{6}$ The lower the concentration of vitamin D in the second trimester, the higher the serum triglyceride (TG) or total cholesterol (TC). ${ }^{7}$ Vitamin D deficiency is prevalent in pregnant women, and it is reported that $47 \%$ of Asian 
pregnant women have a severe deficiency of vitamin D. ${ }^{1}$ Vitamin D deficiency is also common among pregnant women in China. The TG/high-density lipoprotein cholesterol (HDL-c) ratio may be a good marker to identify insulin-resistant Chinese individuals ${ }^{4}$ and is positively associated with diabetes risk. ${ }^{8}$

At present, there is no consistent conclusion about the correlation between vitamin $\mathrm{D}$ and lipid indices. The aim of our research was to investigate whether 25-hydroxyvitamin D $(25(\mathrm{OH}) \mathrm{D})$ status at the second trimester was associated with blood lipids and pregnancy outcomes in patients with GDM.

\section{MATERIALS AND METHODS \\ Participants}

This trial recruited pregnant women from the affiliated Drum Tower Hospital of Nanjing University Medical College in Nanjing, China, between June 2015 and December 2017. The majority $(75 \%-85 \%)$ of pregnant women living in this area (latitude of $32^{\circ} \mathrm{N}$ ) attend the Drum Tower Hospital for antenatal care. Pregnant women who attended the Clinical Nutrition Department of the Drum Tower Hospital were eligible to participate in this trial based on the study criteria.

The inclusion criteria were as follows: (1) age 20-40 years; (2) a plan to visit regularly for prenatal examination and give birth in the Drum Tower Hospital; and (3) a diagnosis of GDM at 24-28 weeks. The exclusion criteria were as follow: (1) unknown data of last menstrual period; (2) twin or multiple pregnancy; (3) abortions $\geq 3$; (4) received assisted reproductive technology; (5) medical or surgical diseases; (6) syphilis, Hepatitis B virus or HIV carrier; (7) a diagnosis of gestational complications (except GDM); (8) previous diagnosis of gestational diabetes (or diabetes); (9) the use of insulin therapy or hypoglycaemic drugs and (10) alcohol abuse, smoking and long-term medication.

We used a self-administered questionnaire and the prenatal maternity card of these participants to collect the basic data (age, height, weight, educational attainment, socioeconomic stats, etc). Of the eligible pregnant women, 261 women were enrolled in the final study.

\section{Assessment of GDM and vitamin D deficiency}

We included pregnant women who were subjected to a $75 \mathrm{~g}$ oral glucose tolerance test (OGTT) at 24-28 weeks. According to the International Association of Diabetes and Pregnancy Study Groups Recommendations guidelines, the diagnosis of GDM was based on the $75 \mathrm{~g}$ OGTT. GDM was diagnosed if the OGTT results equalled or exceeded the following values: fasting plasma glucose $\geq 5.3 \mathrm{mmol} / \mathrm{L}$, OGTT 1 hour $\geq 10.0 \mathrm{mmol} / \mathrm{L}$ and OGTT 2 hour $\geq 8.6 \mathrm{mmol} / \mathrm{L}^{9}$

As a part of the general care of GDM pregnant woman, total serum $25(\mathrm{OH}) \mathrm{D}$ levels are routinely measured in the second trimester after were GDM diagnosis. According to the Endocrine Society Clinical Practice Guideline, vitamin
D deficiency is defined as $25(\mathrm{OH}) \mathrm{D}$ of $<20 \mathrm{ng} / \mathrm{mL}$, insufficiency as $25(\mathrm{OH}) \mathrm{D}$ of $20-30 \mathrm{ng} / \mathrm{mL}$ and sufficiency as $25(\mathrm{OH}) \mathrm{D}$ of $\geq 30 \mathrm{ng} / \mathrm{mL} .{ }^{10}$ Pregnant women with serum $25(\mathrm{OH}) \mathrm{D}$ concentration $<20 \mathrm{ng} / \mathrm{mL}$ were classified into the G1 group $(\mathrm{n}=113)$, and those with serum $25(\mathrm{OH})$ D concentration $\geq 20 \mathrm{ng} / \mathrm{mL}$ were classified into the G2 group ( $\mathrm{n}=148)$.

\section{Pregnancy outcomes and neonatal outcomes}

The clinical outcomes of mothers included gestational weeks of birth and delivery mode. The neonatal outcomes, including birth weight $(\mathrm{g})$ and body length $(\mathrm{cm})$, were retrospectively collected from birth records.

Informed consent is waived by the ethics committee because of the retrospectively study design.

\section{Statistical analysis}

We compared lipid levels and pregnancy outcomes between the two groups ( $n=113$ in the G1 group and $n=148$ in the G2 group). The normality of the variables was visually inspected. Continuous variables were compared using the Mann-Whitney U-test or Kruskal-Wallis $\mathrm{H}$ test, while categorical variables were compared using the $\chi^{2}$ test. The measurement data were expressed as the mean \pm SD $(\bar{x} \pm s)$, and skewed variables were expressed as median (25th percentile and 75th percentile). Moreover, Spearman's correlation analysis and multiple linear regression analysis were used to evaluate the relationship between $25(\mathrm{OH}) \mathrm{D}$ and blood lipids. The relevant confounding variables controlled for the multiple regression analysis included age, height, pre-pregnancy body mass index (BMI), parity, blood pressure, socioeconomic status, educational attainment and season of blood sampling, and $95 \%$ CIs were calculated. $\mathrm{P}<0.05$ was considered statistically significant. Statistical analyses were performed using SPSS software V.22.0.

\section{RESULTS}

Of the eligible pregnant women, 441 patients who met the inclusion criteria were enrolled. The following patients were excluded: 92 patients did not complete the nutrition-related examinations; and 88 patients missed the normal time of antenatal care. In total, 261 women were included in the final population (online supplemental figure 1).

According to $25(\mathrm{OH}) \mathrm{D}$ levels, we divided the patients into G1 and G2 groups. In this study, the mean age of pregnant women with GDM was $30.1 \pm 4.0$ years old, and the mean concentration of serum $25(\mathrm{OH}) \mathrm{D}$ was $22.2 \pm 9.0 \mathrm{ng}$ / $\mathrm{mL}$. Vitamin D deficiency in this population accounted for $43.1 \%(25(\mathrm{OH}) \mathrm{D}<20 \mathrm{ng} / \mathrm{mL})$, and $17.6 \%$ women had sufficient concentrations of $25(\mathrm{OH}) \mathrm{D}$ (table 1 ).

The groups were generally comparable across the background variables, and there were no statistically significant differences in age $(p=0.228)$, height $(p=0.307)$, weight $(\mathrm{p}=0.919)$, pre-pregnancy BMI $(\mathrm{p}=0.588)$ and gravida status $(\mathrm{p}=0.682)$ between the two groups. There 
Table 1 Characteristics of pregnant women according to $25(\mathrm{OH}) \mathrm{D}$ levels

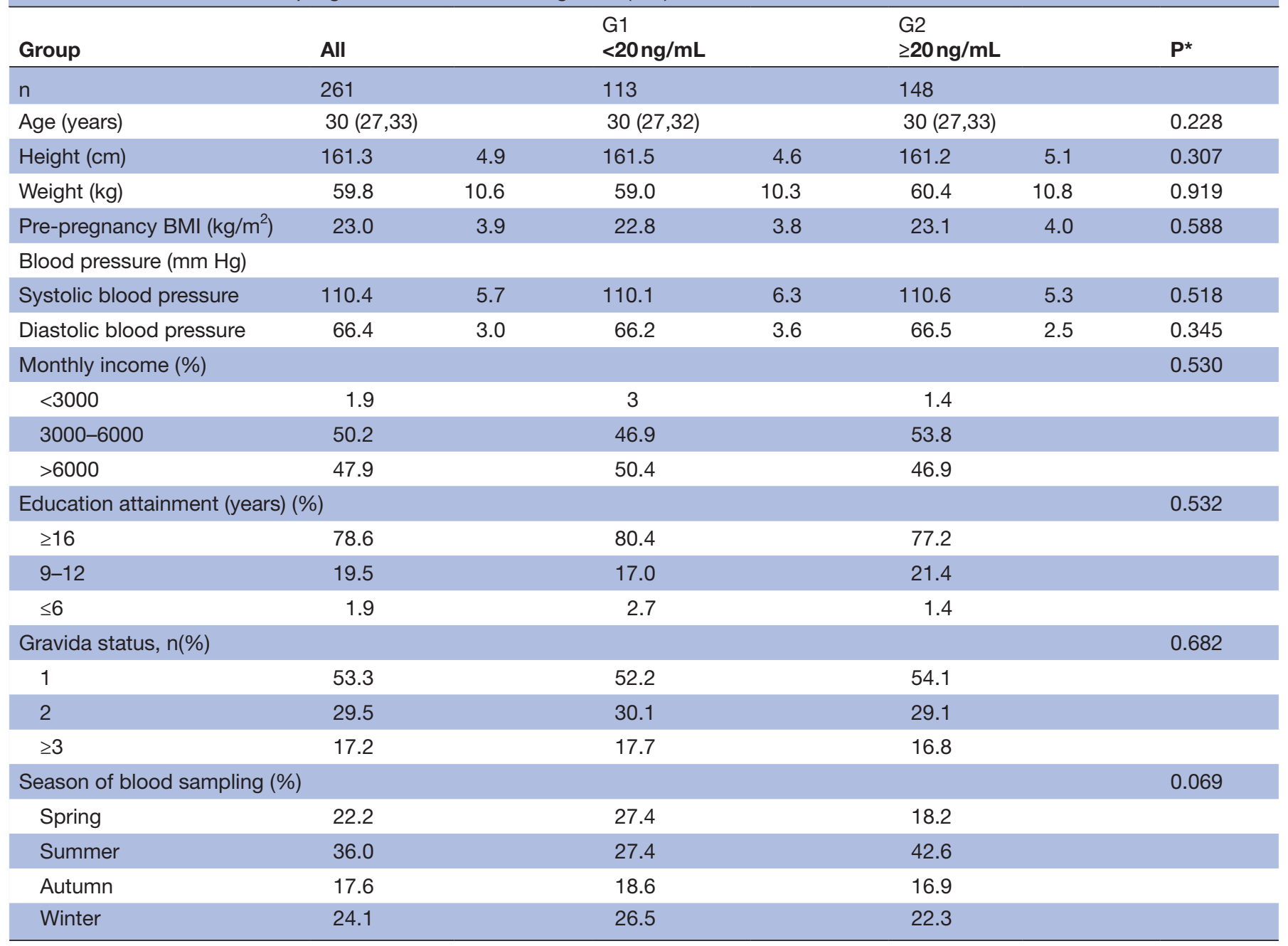

${ }^{*} \mathrm{P}$ values refer to differences between the groups. Normally distributed variables were expressed as mean \pm SD. Skewed variables were expressed as median (25th percentile, 75th percentile).

$\mathrm{BMI}$, body mass index.

was no significant difference in $25(\mathrm{OH}) \mathrm{D}$ levels between women with different educational attainment $(p=0.532)$ and socioeconomic status $(\mathrm{p}=0.530)$. Based on the season of blood sampling, we divided the seasons into spring (from March to May), summer (from June to August), autumn (from September to November) and winter (from December to February). We observed that the prevalence of maternal vitamin D deficiency $(25(\mathrm{OH}) \mathrm{D}$ $<20 \mathrm{ng} / \mathrm{mL}$ ) was higher in winter and lower in summer (table 1).

The results showed that mothers with higher levels of vitamin $\mathrm{D}$ had higher TC $(\mathrm{p}=0.006)$, HDL-c $(\mathrm{p}=0.046)$ and low-density lipoprotein cholesterol (LDL-c) $(p=0.015)$. The TG/HDL-c ratio was significantly higher in the $25(\mathrm{OH}) \mathrm{D}$ deficiency group $(\mathrm{p}=0.035)$. No statistically significant difference was observed between the two groups in terms of TG and TC/HDL-c (table 2).

Regarding the pregnancy outcomes and newborn data, differences between the groups were not significant ( $>0.05$; table 3$)$.
Spearman's correlation analysis between vitamin $\mathrm{D}$ and lipid indices showed that the concentration of $25(\mathrm{OH})$ $\mathrm{D}$ in GDM patients was negatively correlated with TG $(\mathrm{r}=-0.146, \mathrm{p}=0.018)$ and TG/HDL-c $(\mathrm{r}=-0.185, \mathrm{p}=0.003)$ but positively correlated with TC $(\mathrm{r}=0.150, \mathrm{p}=0.016)$ and HDL-c $(r=0.197, \mathrm{p}=0.001)$.

Separate linear regression analysis revealed that $25(\mathrm{OH}) \mathrm{D}$ had a positive correlation with TC, HDL-c and $\mathrm{TG} / \mathrm{HDL}-\mathrm{c}$ ratios $(\mathrm{p}<0.05$ for all). After adjusting for maternal age and pre-pregnancy BMI, 25(OH)D had a positive correlation with HDL-c ( $\mathrm{p}=0.046$; model 1 , table 4). However, after including socio-economic status in the models, maternal $25(\mathrm{OH}) \mathrm{D}$ was no longer associated with HDL-c. In models with additional adjustment for parity, educational attainment, height, blood pressure, socioeconomic status, season and gestational age, maternal $25(\mathrm{OH}) \mathrm{D}$ was inversely associated with TG/HDL-c ratios $(\mathrm{B}=-0.016 ; 95 \% \mathrm{CI}=-0.025$ to 0.006 ; model 2, table 4). Although the model of TG/HDL-c 
Table 2 Lipid profile indicators and vitamin D levels in the same period

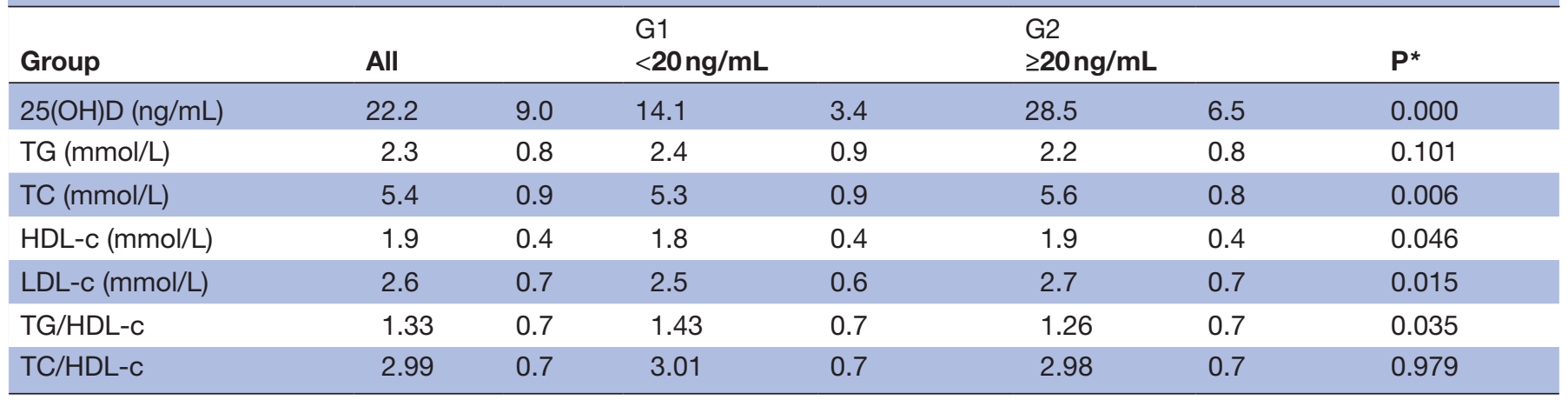

Normally distributed variables were expressed as mean \pm SD.

${ }^{*} \mathrm{P}$ values refer to differences between the groups.

HDL-c, high-density lipoprotein cholesterol; LDL-c, low-density lipoprotein cholesterol; 25(OH)D, 25-hydroxyvitamin D; TC, total cholesterol; TG, triglyceride.

and $25(\mathrm{OH}) \mathrm{D}$ was statistically significant $(\mathrm{p}=0.036)$, the model was not sufficiently interpreted $\left(\mathrm{R}^{2}=6.9 \%\right)$.

\section{DISCUSSION}

In this study, we found a high incidence of vitamin D deficiency in pregnant women with GDM. After adjusting for age, pre-pregnancy BMI, parity, height, blood pressure, socioeconomic status, educational attainment, season and gestational age, maternal $25(\mathrm{OH}) \mathrm{D}$ at 24-28 weeks was inversely associated with TG/HDL-c ratios. Moreover, there was no relationship between vitamin $\mathrm{D}$ and pregnancy outcomes, newborn weight and newborn length.

In the presentstudy, maternal 25(OH)D in GDM patients was negatively correlated with triglycerides $(\mathrm{r}=-0.146)$ and TG/HDL-c $(\mathrm{r}=-0.185)$ while positively correlated with TC $(r=0.150)$ and HDL-c $(r=0.197)$, which was consistent with previously reported results for maternal $25(\mathrm{OH}) \mathrm{D}$ and blood lipid-related indicators. ${ }^{11}$ However, the present results contradicted the results reported by Lepsch, who studied the relationship research between vitamin $\mathrm{D}$ and TC. ${ }^{12}$ This difference may be related to the selection of GDM population. In addition, pregnant women have a certain degree of physiological increase of TC. There were no statistically significant differences in gestational weeks and caesarean section rate between the two groups, which disagreed with the research reported by Merewood et $a l .{ }^{13}$ There was no independent relationship between vitamin D concentration and newborn outcomes, which was consistent with the research results of a multi-ethnic population-based study ${ }^{14}$ and a meta-analysis. ${ }^{15}$ Vitamin D levels have been shown to correlate with blood glucose and lipid index in the first trimester. ${ }^{9}$ In our city, early pregnancy tests are generally performed at community hospitals. Considering that the community measurement methods are not unified and data collection is incomplete, we did not conduct the relevant research. Thus, we were unable to verify the changes of the pregnancy.

During the entire pregnancy, the physiological state and metabolism of pregnant women is greatly changed, especially the endocrine system and insulin secretion. Increased levels of insulin in the circulatory system lead to abnormal glucose tolerance of pregnant women and an increased incidence of gestational diabetes. In recent years, several studies have found that vitamin $\mathrm{D}$ acts as a hormone. Studies have shown that low vitamin D concentration affects glycaemic control in patients with gestational diabetes. ${ }^{816}$ Vitamin $\mathrm{D}$ is a steroid derivative that is mainly stored in adipose tissue. Investigation of the effect of vitamin $\mathrm{D}$ intervention on glucose and lipid metabolism in patients with type 2 diabetes ${ }^{17}$ has shown that vitamin $\mathrm{D}$ affects glucose and lipid metabolism in patients.

In patients with diabetes and insulin resistance, dyslipidaemia is characterised by increased TG levels and decreased HDL-c levels. ${ }^{18}$ Studies have shown that higher

Table 3 Pregnancy outcomes and newborn data

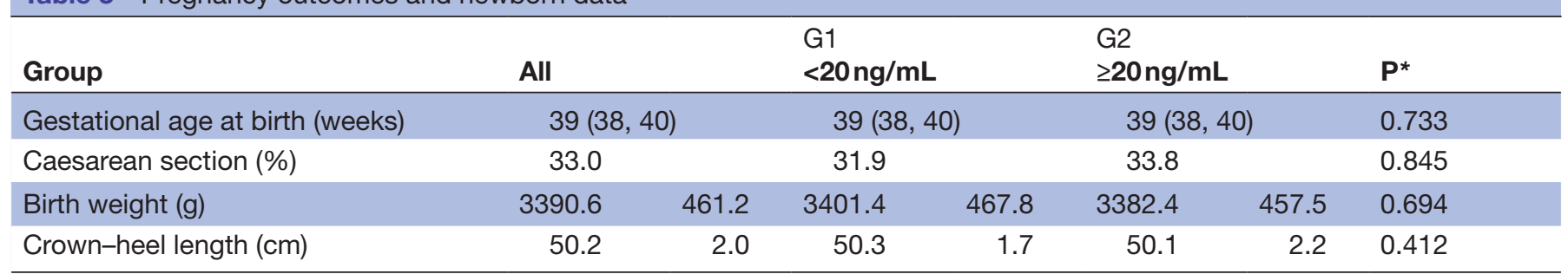

Normally distributed variables were expressed as mean \pm SD, skewed variables were expressed as median. $\left(25^{\text {th }}\right.$ percentile, $75^{\text {th }}$ percentile). ${ }^{*} P$ values refer to differences between the groups. 
serum TG or TC levels in the second trimester result in lower patient's $25(\mathrm{OH}) \mathrm{D}$ concentrations, which may lead to the higher risk of GDM. ${ }^{7120}$ This phenomenon may provide the basis for early prediction, diagnosis and treatment of GDM, thereby reducing the risk of adverse pregnancy outcomes. ${ }^{21}$ The TG/HDL-c ratio may be a good marker to identify insulin-resistant Chinese individuals ${ }^{4}$ and is positively associated with diabetes risk. ${ }^{22}{ }^{23}$ Our finding about TG/HDL-c ratios implied that it may be possible to decrease the TG/HDL-c ratio by increasing the concentration of $25(\mathrm{OH}) \mathrm{D}$, which would be helpful for improving insulin resistance. In healthy normal pregnancies, we have previously observed that inadequate $25(\mathrm{OH}) \mathrm{D}$ concentrations during early pregnancy are associated with more pronounced changes of TC, LDL-c concentrations and TC/HDL-c ratios throughout pregnancy. ${ }^{12}$ Previous study results are contradictive and do not clearly support therapeutic supplementation as a safe strategy for the prevention of pregnancy complications. ${ }^{23}$ However, considering there was a high prevalence of vitamin D insufficiency in our study, we recommend that all pregnant women, especially those with GDM, be regularly tested and prompt treatment for women who are vitamin D deficient.

To the best of our knowledge, this is the first study to evaluate the association between $25(\mathrm{OH}) \mathrm{D}$ concentrations at 24-28 weeks and lipid profile during pregnancy in a GDM group in the Chinese population. In this study, we designed a questionnaire containing clinically relevant factors, including age, BMI, parity and seasons, for women at different educational attainment, and we trained students to help the pregnant women fill out the questionnaire. Serum 25(OH)D level was measured after women were diagnosed with GDM, which enabled assessment of longitudinal relationships between $25(\mathrm{OH}) \mathrm{D}$ and pregnancy outcomes in the GDM population. There were several limitations in this study. First, the sample size of this study was insufficient. We did not include healthy normal pregnant women due to the small sample size of healthy pregnant women participating in regular vitamin D checks, which had a certain impact on the results and could not explain the relationship between vitamin $\mathrm{D}$ levels and the incidence of GDM. ${ }^{24}$ Second, our research measured total vitamin D. Measurements of free vitamin D might be more appropriate due to the stimulation of vitamin D-binding protein during pregnancy. Tsuprykov et al found that free $25(\mathrm{OH}) \mathrm{D}$ revealed better associations with markers of lipid metabolism. ${ }^{25}$ Free vitamin D measurements are independent of confounding factors, such as pregnancy and genetic background, and may be much better correlated with the lipids and clinical outcome.${ }^{26}$ Third, in the process of data collection, the time that some pregnant women had serum $25(\mathrm{OH}) \mathrm{D}$ levels measured was not consistent with the biochemical examination time (differences up to 2 weeks), which may have led to numerical differences, thereby affecting the interpretation of the model.

In summary, our study found that HDL-c levels and TG/ HDL-c ratios in patients with gestational diabetes during 
the second trimester may be affected by serum $25(\mathrm{OH})$ $\mathrm{D}$ levels. It is necessary to perform regular testing and prompt treatment for women with GDM who are vitamin D deficient. The present research provided a foundation for future studies that aim to investigate the benefits of controlling blood lipids in pregnant women with GDM. Thus, further observational studies and interventional randomised controlled trials are still needed to confirm the association between blood lipids and $25(\mathrm{OH}) \mathrm{D}$ of GDM patients.

Acknowledgements We thank all the patients enrolled in the study, and staff of clinical nutrition department in affiliated Drum Tower Hospital of Nanjing University Medical College.

Contributors WC and XC designed the study. WC collected the data and wrote the manuscript. WC, YL and XC analysed and interpreted the data. YL and BG helped in English editing and revised the article. JL and MZ helped in clinical data collection and procedure implementation. All authors read and approved the final version of the manuscript submitted for publication.

Funding This study was funded by the Key Project supported by Medical Science and Technology Development Foundation, Nanjing Department of Health (YKK16082, YKK17087 and YKK18079).

Competing interests None declared.

Patient consent for publication Obtained.

Ethics approval We performed an observational cohort study. The study was approved by the Clinical Research Ethics Committee of the Nanjing Drum Tower Hospital, the affiliated hospital of Nanjing University Medical School. All the data were collected retrospectively and analysed anonymously. Informed consent is waived by the ethics committee because of the retrospectively study design. All procedures were performed in accordance with the principles of the Declaration of Helsinki.

Provenance and peer review Not commissioned; externally peer reviewed.

Data availability statement All data relevant to the study are included in the article or uploaded as supplementary information.

Supplemental material This content has been supplied by the author(s). It has not been vetted by BMJ Publishing Group Limited (BMJ) and may not have been peer-reviewed. Any opinions or recommendations discussed are solely those of the author(s) and are not endorsed by BMJ. BMJ disclaims all liability and responsibility arising from any reliance placed on the content. Where the content includes any translated material, BMJ does not warrant the accuracy and reliability of the translations (including but not limited to local regulations, clinical guidelines, terminology, drug names and drug dosages), and is not responsible for any error and/or omissions arising from translation and adaptation or otherwise.

Open access This is an open access article distributed in accordance with the Creative Commons Attribution Non Commercial (CC BY-NC 4.0) license, which permits others to distribute, remix, adapt, build upon this work non-commercially, and license their derivative works on different terms, provided the original work is properly cited, appropriate credit is given, any changes made indicated, and the use is non-commercial. See: http://creativecommons.org/licenses/by-nc/4.0/.

\section{ORCID iD}

Xiaotian Chen http://orcid.org/0000-0001-6112-0784

\section{REFERENCES}

1 Yuen L, Saeedi P, Riaz M, et al. Projections of the prevalence of hyperglycaemia in pregnancy in 2019 and beyond: results from the International diabetes Federation diabetes atlas, 9th edition. Diabetes Res Clin Pract 2019;157:107841.

2 Herrera E, Ortega-Senovilla H. Lipid metabolism during pregnancy and its implications for fetal growth. Curr Pharm Biotechnol 2014;15:24-31.

3 Lapillonne A. Vitamin D deficiency during pregnancy may impair maternal and fetal outcomes. Med Hypotheses 2010;74:71-5.
4 Gasevic D, Frohlich J, Mancini GBJ, et al. The association between triglyceride to high-density-lipoprotein cholesterol ratio and insulin resistance in a multiethnic primary prevention cohort. Metabolism 2012;61:583-9.

5 Rodriguez A, García-Esteban R, Basterretxea M, et al. Associations of maternal circulating 25-hydroxyvitamin D3 concentration with pregnancy and birth outcomes. BJOG 2015;122:1695-704.

6 Black LJ, Burrows S, Lucas RM, et al. Serum 25-hydroxyvitamin D concentrations and cardiometabolic risk factors in adolescents and young adults. Br J Nutr 2016;115:1994-2002.

7 Chen J, Cheng W, Zhao G, et al. Serum 25-hydroxyvitamin D and serum lipid levels in pregnant women with GDM and their clinical significance. Maternal and Child Health Care of China 2017;32:4649-51.

8 El Lithy A, Abdella RM, El-Faissal YM, et al. The relationship between low maternal serum vitamin $D$ levels and glycemic control in gestational diabetes assessed by $\mathrm{HbA} 1 \mathrm{c}$ levels: an observational cross-sectional study. BMC Pregnancy Childbirth 2014;14:362.

$9 \mathrm{Hu}$ L, Zhang $\mathrm{Y}$, Wang $\mathrm{X}$, et al. Maternal vitamin D status and risk of gestational diabetes: a meta-analysis. Cell Physiol Biochem 2018:45:291-300.

10 Holick MF, Binkley NC, Bischoff-Ferrari HA, et al. Evaluation, treatment, and prevention of vitamin D deficiency: an endocrine Society clinical practice guideline. J Clin Endocrinol Metab 2011;96:1911-30.

11 Maki KC, Rubin MR, Wong LG, et al. Serum 25-hydroxyvitamin D is independently associated with high-density lipoprotein cholesterol and the metabolic syndrome in men and women. $J$ Clin Lipidol 2009;3:289-96

12 Lepsch J, Eshriqui I, Farias DR, et al. Association between early pregnancy vitamin $\mathrm{D}$ status and changes in serum lipid profiles throughout pregnancy. Metabolism 2017;70:85-97.

13 Merewood A, Mehta SD, Chen TC, et al. Association between vitamin D deficiency and primary cesarean section. J Clin Endocrinol Metab 2009;94:940-5

14 Eggemoen Åse R, Jenum AK, Mdala I, et al. Vitamin D levels during pregnancy and associations with birth weight and body composition of the newborn: a longitudinal multiethnic population-based study. $\mathrm{Br}$ J Nutr 2017; 117:985-93.

15 Thorne-Lyman A, Fawzi WW. Vitamin D during pregnancy and maternal, neonatal and infant health outcomes: a systematic review and meta-analysis. Paediatr Perinat Epidemiol 2012;26 Suppl 1:75-90.

16 Lau SL, Gunton JE, Athayde NP, et al. Serum 25-hydroxyvitamin D and glycated haemoglobin levels in women with gestational diabetes mellitus. Med J Aust 2011;194:334-7.

17 Niroomand M, Fotouhi A, Irannejad N, et al. Does high-dose vitamin $D$ supplementation impact insulin resistance and risk of development of diabetes in patients with pre-diabetes? A double-blind randomized clinical trial. Diabetes Res Clin Pract 2019;148:1-9.

18 Quispe R, Manalac RJ, Faridi KF, et al. Relationship of the triglyceride to high-density lipoprotein cholesterol (TG/HDL-C) ratio to the remainder of the lipid profile: the very large database of Lipids-4 (VLDL-4) study. Atherosclerosis 2015;242:243-50.

19 Zhang Y, Gong Y, Xue H, et al. Vitamin D and gestational diabetes mellitus: a systematic review based on data free of Hawthorne effect. BJOG 2018;125:784-93.

20 Eslami B, Naddafi K, Rastkari N, et al. Association between serum concentrations of persistent organic pollutants and gestational diabetes mellitus in primiparous women. Environ Res 2016;151:706-12.

21 Lu L, Bennett DA, Millwood IY, et al. Association of vitamin D with risk of type 2 diabetes: a Mendelian randomisation study in European and Chinese adults. PLoS Med 2018;15:e1002566.

22 Chen Z, Hu H, Chen M, et al. Association of triglyceride to highdensity lipoprotein cholesterol ratio and incident of diabetes mellitus: a secondary retrospective analysis based on a Chinese cohort study. Lipids Health Dis 2020;19.

23 von Websky K, Hasan AA, Reichetzeder C, et al. Impact of vitamin D on pregnancy-related disorders and on offspring outcome. J Steroid Biochem Mol Biol 2018;180:51-64.

24 Zhou J, Liu C, Zhao N. Serum 25-hydroxyvitamin D_3 and serum lipid levels in patients with gestational diabetes mellitus in the second trimester and its role in the pathogenesis [J]. Maternal and Child Health Care of China 2017;32:4657-9.

25 Tsuprykov O, Buse C, Skoblo R, et al. Comparison of free and total 25 -hydroxyvitamin $D$ in normal human pregnancy. J Steroid Biochem Mol Biol 2019;190:29-36.

26 Tsuprykov O, Chen X, Hocher C-F, et al. Why should we measure free 25(OH) vitamin D? J Steroid Biochem Mol Biol 2018;180:87-104. 